\title{
Impact on outcomes after listing and transplantation, of a strategy to accept ABO blood group-incompatible donor hearts for neonates and infants
}

Lori J. West, MD, DPhil, Tara Karamlou, MD, Anne I. Dipchand, MD, Stacey M. Pollock-BarZiv, PhD, John G. Coles, MD, and Brian W. McCrindle, MD, MPH

From the Division of Cardiology, Department of Pediatrics, The Hospital for Sick Children, University of Toronto, Toronto, Ontario, Canada.

Presented, in part, at the American Heart Association Annual Scientific Sessions, Orlando, Fla, November 2003.

Received for publication July 19, 2005; revisions received Sept 6, 2005; accepted for publication Sept 15, 2005.

Address for reprints: Brian W. McCrindle, MD, The Hospital for Sick Children, 555 University Ave, Toronto, Ontario, Canada M5G 1X8 (E-mail: brian.mccrindle@ sickkids.ca).

J Thorac Cardiovasc Surg 2006;131:455-61

$0022-5223 / \$ 32.00$

Copyright () 2006 by The American Association for Thoracic Surgery

doi:10.1016/j.jtcvs.2005.09.048
Background: Recent data suggest that $\mathrm{ABO}$ blood group-incompatible donor hearts are immunologically well tolerated in infants undergoing transplantation.

Methods: Competing-risks methodology was used to assess outcomes after listing and the impact of a strategy to accept heart grafts from any blood group donor for infants less than 18 months of age.

Results: From 1992 to 2002, there were 91 listing episodes in 84 patients (including 20 fetuses; $50 \%$ were male and $63 \%$ had congenital heart disease). Beginning in 1995, a strategy to accept ABO-incompatible organs was adopted. Competing-risks analysis showed that after 20 months $60 \%$ underwent transplantation, $18 \%$ died, and less than $1 \%$ were still listed; the remaining $21 \%$ were de-listed because of a change of surgical strategy (9\%), improved clinical condition (8\%), and deterioration to ineligibility (4\%). Risk factors for transplantation included only a strategy to accept ABO-incompatible organs $(P<.001)$. Risk factors for death included failure to accept ABO-incompatible organs $(P=.002)$ and Canadian listing status $3(P=.085)$ or $4(P<.001)$. Multivariable parametric models were used to create competing risk predictions for outcomes specific to status and ABO-incompatible strategy. Higher status resulted in greater mortality regardless of strategy, although for any status, more patients underwent transplantation and fewer died using a strategy to accept ABO-incompatible organs. Parametric modeling of time-related freedom from death or retransplantation demonstrated no significant difference at 4 years posttransplantation $(P=.78)$ for $\mathrm{ABO}$-incompatible $(74 \%)$ versus $\mathrm{ABO}$-compatible transplants $(72 \%)$.

Conclusions: A strategy to accept ABO-incompatible donor hearts for infant transplantation significantly improves the likelihood of transplantation and reduces waiting list mortality while not adversely altering outcomes after transplantation.

$\mathrm{S}$ ince the early days of organ transplantation, $\mathrm{ABO}$ blood group compatibility between donors and recipients has been understood to be mandatory because of the high risk of "hyperacute" rejection. ${ }^{1-3}$ This process occurs when preexisting recipient "natural" antibodies (isoagglutinins) directed against donor A and/or B blood groups bind their cognate antigens on donor organ endothelium. A rapidly progressive cascade is initiated with complement activation and recruitment of other inflammatory mediators, leading to irreversible and widespread thrombosis of graft vasculature. Because of the chronic shortage of donor organs, attempts have been made to bridge the blood group barrier, with some success in kidney and liver transplantation. ${ }^{3-10}$ These attempts have required aggressive strategies to remove antidonor antibody, including splenectomy, plasmapheresis, and immunoadsorption, but antibody often reaccumulates because of B-cell memory combined with antigen persistence. 


\section{Abbreviations and Acronyms \\ $\mathrm{ABO}-\mathrm{I}=\mathrm{ABO}$ incompatible}

Heart transplantation across $\mathrm{ABO}$ incompatibility is never undertaken intentionally in adults because of the high lethality rate of the few reported accidental cases. ${ }^{11} \mathrm{We}$ previously reported that the requirement for $\mathrm{ABO}$ compatibility in heart transplantation seems to be unnecessary for young infants because of their natural delay in development of humoral immunity to T-independent antigens, including isoagglutinin production. ${ }^{12}$ In the present study we sought to determine the impact on outcomes, after listing and transplantation, of adopting a strategy to accept ABO-incompatible (ABO-I) donor hearts for infants.

\section{Methods}

\section{Patient Characteristics}

From computerized databases, all infants less than 18 months of age at listing from 1992 to 2002 were identified. All patients were cared for and underwent transplantation at the Hospital for Sick Children. Data regarding patient characteristics and outcomes were collected. Historical patients from the same institution were compared with patients who were listed with an ABO-I strategy and who underwent an ABO-I transplantation in an unmatched comparison.

We identified 91 listing episodes in 84 patients. Fifty percent of the patients were male, and $63 \%$ had congenital heart disease, with $48 \%$ having hypoplastic left heart syndrome. ABO blood group was group O in 41 patients (49\%), group A in 33 patients (39\%), group $\mathrm{B}$ in 8 patients (10\%), and group $\mathrm{AB}$ in 2 patients (4\%). Twenty patients were initially listed in utero. Of the remaining episodes, the median age at listing was 2.7 months, ranging from birth to 18 months. Seven listing episodes occurred while the patient was on extracorporeal membrane oxygenation. The status at listing (Canadian status system) was 3.5 to 4 (most urgent) in $41 \%, 3$ in $44 \%$, and 1 (least urgent) or 2 in $15 \%$ (Table 1).

Beginning in 1995 a strategy to accept ABO-I donor hearts was adopted, meaning that all infants less than 18 months of age were listed for any donor blood group rather than indicated for a specific donor blood group, as had been our previous strategy. All infants less than 18 months of age were assessed for appropriateness for listing for ABO-I heart transplantation. The decision to list a patient for a potential donor of any blood group was based on the (1) absence or low level of preformed isohemagglutinins and (2) parental consent. No parents refused to accept an ABO-I graft during the study period. Patients of any status level were eligible, and therefore patients were not excluded from this listing strategy if they were "too well." Organs were allocated by the established method in Canada, taking into consideration listing status and geographic location. During the course of the study, 20 donor organs were procured from the United States. There was no separate allocation algorithm for patients listed for ABO-I transplantation (ie, the organ was not offered to an ABO-I recipient only if there was no compatible recipient). Organs were accepted on the basis of the standard decision-making criteria taking into account both donor and recipient factors. Blood group was not factored into the decision making (ie, a patient on the ABO-I list received the first good-quality organ, and organs were not declined on the basis of blood group incompatibility even if the recipient was stable).

Overall, $76 \%$ of listing episodes (69 listing episodes in 65 patients) included in the study were made with an ABO-I strategy. Sixteen patients with an ABO-I listing eventually underwent transplantation with ABO-I hearts. Thus, the majority of patients (75\%) listed with the ABO-I strategy still received ABO-compatible organs.

\section{Statistical Analysis}

Data are described as frequencies, medians with ranges, and means with standard deviations as appropriate. To determine the impact of the ABO-I blood group strategy on outcomes after listing, the following mutually exclusive time-related states were defined: death, achievement of transplantation, removal from the list, and ongoing survival without transition to another state. In addition, the state of having been removed from the list was further defined according to the following reasons: the patient's clinical status improved such that transplantation was no longer necessary, the patient's clinical status deteriorated such that he or she was no longer considered a candidate for transplantation (eg, multiorgan failure), or the patient had an alternative procedure (eg, Norwood Stage I operation for hypoplastic left heart syndrome). The rates of transition from the initial state to each competing state were determined, and these were synthesized in a competing-risks analysis to give the proportion of listed patients achieving each state at any given time after listing. ${ }^{13}$ Risk-unadjusted nonparametric estimates of time-related freedom from each state were plotted as Kaplan-Meier estimates. Calculation of parametric estimates of time-related freedom from each state was achieved by modeling the hazard function and determining the characteristic equation for each phase of risk. ${ }^{14}$ Factors associated with each state, especially use of the ABO-I blood group strategy, were sought in multivariable hazard analysis in the totally parametric domain. Factors associated with 5 or fewer events were not considered to minimize the risk of model overdetermination. Bootstrap bagging was used to guide final factor selection as previously described..$^{15}$ The multivariable models were then solved in a competing-risks analysis to demonstrate the magnitude of effect of significant factors on the proportion of patients transitioning to all states. In addition, factors associated with time-related outcomes after transplantation were also sought. Because events were infrequent, the major timerelated outcomes of death and retransplantation were combined, and an analysis similar to that previously described was performed without the competing-risks component.

\section{Results \\ Events After Listing}

Mortality. Time-related death after listing occurred for 16 patients (Figure 1, $A$ ). There was a gradual early hazard or risk phase, with an ongoing risk of death up to 20 months after listing. Incremental risk factors for death included status 3 or 4 (more "ill") at time of listing $(P<.001$, bootstrap reliability $91 \%$ ) and failure to use the ABO-I 
TABLE 1. Canadian status at listing compared with United Network for Organ Sharing

\begin{tabular}{|c|c|}
\hline Canadian status (no separate pediatric listing policy like UNOS) & UNOS pediatric patient status \\
\hline 1: Waiting at home & 2: Patient not meeting criteria for status $1 \mathrm{~A}$ or $1 \mathrm{~B}$ \\
\hline \multicolumn{2}{|l|}{ 2: Hospitalized with complication of heart disease } \\
\hline 3: Ward care + VAD or inotropes & 1B: One of the following: \\
\hline 3.5: ICU care + PA catheter + high-dose/multiple IV inotropes & $\begin{array}{l}\text { Low-dose single inotrope, }<6 \text { months old but not } \\
\text { meeting } 1 \mathrm{~A} \text { criteria, growth failure (pediatric) }\end{array}$ \\
\hline \multirow[t]{2}{*}{ 4: ICU care + mechanical ventilatory or circulatory support } & 1A: One of the following: \\
\hline & $\begin{array}{l}\text { Ventilator, mechanical assist device, balloon } \\
\text { pump, high-dose or multiple inotropes, patient } \\
<6 \text { months old with congenital or acquired } \\
\text { heart disease exhibiting reactive pulmonary } \\
\text { hypertension at }>50 \% \text { of systemic level; life } \\
\text { expectancy of }<14 \text { days without a heart } \\
\text { transplant }\end{array}$ \\
\hline
\end{tabular}

$>$ UNOS, United Network for Organ Sharing; VAD, venous access device; ICU, intensive care unit; $P A$, pulmonary artery.

listing strategy $(P<.002$, bootstrap reliability $69 \%)$. No other factors were significantly associated with death without transplantation, including date and age at listing.

Transplantation. Figure 1, $B$ shows the time-related freedom from transplantation, with 54 listing episodes resulting in transplantation. Sixteen patients received ABO-I grafts, and 38 patients received $\mathrm{ABO}$-compatible grafts. ABO-I recipients were similar to those receiving ABO-I grafts (Table 2). There was a single prolonged early hazard or risk phase. The only significant factor predicting transplantation was the use of the ABO-I listing strategy $(P<.001$, bootstrap reliability $86 \%)$.

De-listing. Removal from the list occurred in 20 patients. Reasons for removal included (1) clinical deterioration to ineligibility for transplantation in 4 patients; (2) clinical improvement in 8 patients; and (3) performance of an alternative procedure in 8 patients. Risk factors were not sought for these events because of the small number of patients achieving these less frequent events.

Competing-risks analysis. The majority of events occurred within the first 3 months after listing. The competingrisks depiction of all 6 mutually exclusive end states occurring after listing is shown in Figure 2. Competing-risks analysis predicted that at 20 months after listing, $60 \%$ of the patients had undergone transplantation, $18 \%$ had died while listed, and $21 \%$ had been de-listed. Of those who were de-listed, an alternative procedure was performed in $9 \%$, $8 \%$ were removed from the list because they improved, and $4 \%$ were removed because of clinical deterioration precluding transplantation.

Outcome predictions were generated by solving the multivariable competing-risk equations for the 2 significant incremental risk factors, including status at listing and use of the ABO-I strategy. Use of the ABO-I strategy resulted in
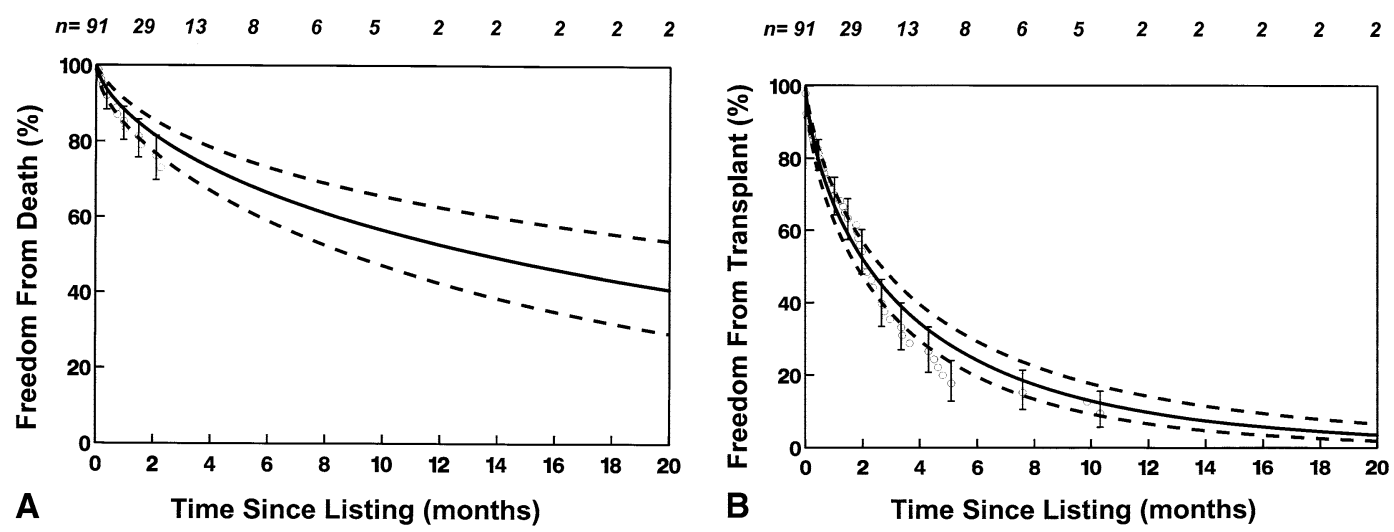

Figure 1. Time-related freedom from outcomes after listing for transplant. A, Time-related freedom from death while listed. B, Time-related freedom from transplantation after listing. Nonparametric Kaplan-Meier estimates (circles with bars). Parametric model of the hazard function (solid line) with $70 \%$ confidence interval (dashed lines). Number of patients at risk (numbers at top of graph). 
TABLE 2. Characteristics of patients who underwent transplantation $(N=54)$

\begin{tabular}{lccc}
\hline \multicolumn{1}{c}{ Variable } & $\begin{array}{c}\text { ABO-I recipients } \\
(\mathbf{n}=\mathbf{1 6})\end{array}$ & $\begin{array}{c}\text { ABO-compatible recipients } \\
(\mathbf{n}=\mathbf{3 8})\end{array}$ & $\boldsymbol{P}$ \\
\hline $\begin{array}{l}\text { Male } \\
\text { Diagnosis }\end{array}$ & $7(43 \%)$ & $17(45 \%)$ & .94 \\
$\quad$ HLHS & $10(63 \%)$ & $13(34 \%)$ & .06 \\
Other CHD & $12(75 \%)$ & $25(66 \%)$ & .50 \\
Pretransplant ECMO & $2(12 \%)$ & $2(5 \%)$ & .26 \\
Median age at listing, mo & $21 \mathrm{~d}$ (in utero-12) & $40 \mathrm{~d}$ (in utero-16) & .25 \\
Median age at transplant, mo & 2 (birth-15 mo) & 3 (birth-30) & .24 \\
\hline
\end{tabular}

$>A B O-I, A B O$-incompatible; HLHS, hypoplastic left heart syndrome; ECMO, extracorporeal membrane oxygenation; $C H D$, congenital heart disease.

important decreases in the proportion of patients who died, regardless of the patient's status at listing (Figure 3,A), and as expected, lower status at listing (less "ill") was also associated with lower mortality. Similarly, use of the ABO-I strategy also resulted in an important increase in the proportion of patients achieving transplantation, regardless of their status at listing, with patients at lower status more likely to achieve transplantation (Figure 3,B).

Figure 4, $A$, shows the time-related freedom from either death $(\mathrm{n}=13)$ or retransplantation $(\mathrm{n}=2)$ after transplantation. There was a steep early hazard phase followed by an ongoing low constant phase of risk. Parametric estimates of freedom from this composite outcome were $73 \%$ at 1 year,

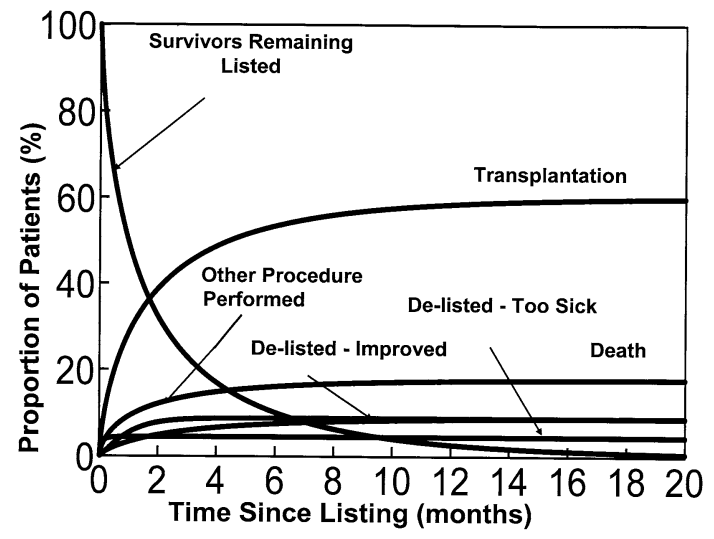

Figure 2. Competing-risks depiction of events after listing for transplant. All patients begin alive and thereafter migrate to 1 of 6 mutually exclusive end states (death, transplantation, de-listing secondary to clinical deterioration, de-listing secondary to improved clinical condition, de-listing secondary to a change in surgical procedure, and remaining alive without transplantation) at a time-dependent rate defined by the underlying hazard functions. At any point in time, the sum of the proportion of children in each state is $100 \%$. For example, the estimated prevalences at 20 months after listing are as follows: transplantation $(60 \%)$, death without transplantation $(18 \%)$, de-listed $(21 \%)$, and alive without transplantation ( $<1 \%$ ). Parametric point estimates (solid lines).
$70 \%$ at 5 years, and $67 \%$ at 8 years after transplantation. Risk factors were sought for this time-related outcome, and none were significant, specifically that of receiving an ABO-I organ (Figure 4, B).

\section{Discussion}

Long-term survival for patients undergoing heart transplantation in early infancy has improved, especially when considering conditional survival beyond 12 months posttransplant. ${ }^{16,17}$ This may be partly because of the ease of manipulating the infant immune response to the transplanted organ, an immunologic advantage that seems most pronounced in neonatal recipients. ${ }^{18}$ However, wider availability of this therapy for infants with otherwise lethal cardiac malformations and cardiomyopathies has been severely limited by the critical shortage of donor organs of both compatible blood group and appropriate size.

The perceived requirement for $\mathrm{ABO}$ compatibility has been demonstrated to be particularly detrimental for infants of blood group $\mathrm{O}$ awaiting transplantation, who face disproportionate competition for blood group $\mathrm{O}$ donors whose organs are compatible with all 4 recipient blood groups. ${ }^{19}$ Moreover, a paucity of data and lack of consensus guidelines for brain death determination in the very young have further exacerbated the scarcity of potential organ donors for the smallest recipients. ${ }^{20}$ Thus, infants have had relatively higher mortality rates while awaiting transplantation than older (and larger) patients. ${ }^{19}$

The stringent requirement for $\mathrm{ABO}$ compatibility in organ transplantation evolved for children by extrapolation from experience with adult patients, rather than as a primary consideration based on appropriate developmental parameters for pediatrics. The rationale for our initial cohort of ABO-I transplants in infants was based on well-established immunologic milestones of childhood coupled with a compelling clinical need to expand the potential pool of organ donors, with the added knowledge that available organs were being wasted because of lack of ABO-compatible recipients. Our initial experience demonstrated that accepting ABO-I donor organs for infant patients during the normal developmental lag in 

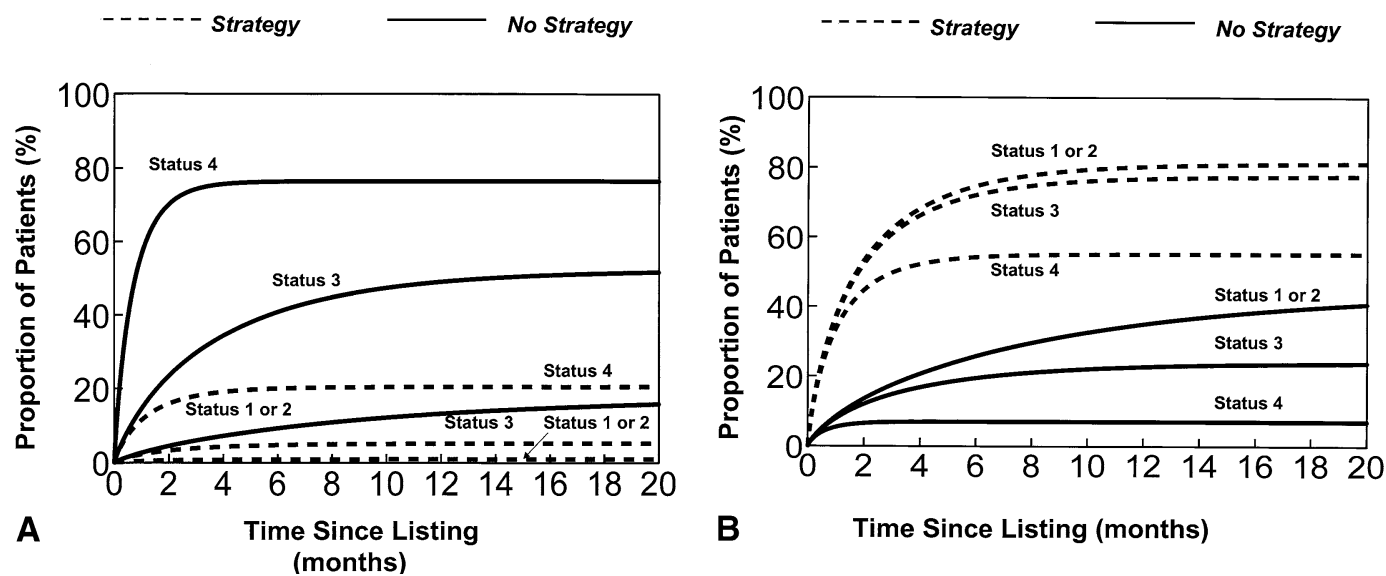

Figure 3. Multivariable competing risks predicting outcomes solved for ABO strategy and status at listing. A, Multivariable competing risks predicting the proportion dying while listed solved for ABO blood group strategy and status at listing. This graph represents specific solutions to the multivariable competing risk equation for death without transplantation using specific values for the patient status $(1$ and 2,3, or 4$)$ and the $A B O$ strategy (ABO-I vs $\mathrm{ABO}$-compatible.) The use of an ABO-I strategy (dashed lines) results in a lower predicted proportion of patients dying without transplantation for all status groups compared with the use of an ABO-compatible strategy (solid lines). B, Multivariable competing risks predicting the proportion achieving transplantation after listing solved for ABO blood group strategy and status at listing. Similarly, this graph represents specific solutions to the multivariable competing-risks equation for the achievement of transplantation using specific values for the patient status (1 and 2, 3, or 4 ) and the $A B O$ strategy (ABO-I vs $A B 0$-compatible.) The use of an ABO-I strategy (dashed lines) results in a greater predicted proportion of patients who underwent transplantation for all status groups compared with the use of an ABO-compatible strategy (solid lines).

humoral immunity was feasible and safe, in the short term at least, as a partial solution for the critical organ donor shortage. In the present study, we examined whether our assessment of the risks and benefits of crossing the $\mathrm{ABO}$ barrier was justified by examining whether adopting a strategy to accept ABO-I donor hearts resulted in better outcomes for infants listed for transplantation.

The only 2 factors we found to be significantly associated with mortality while waiting were higher status (more "ill"), reflecting their more urgent need for timely organ
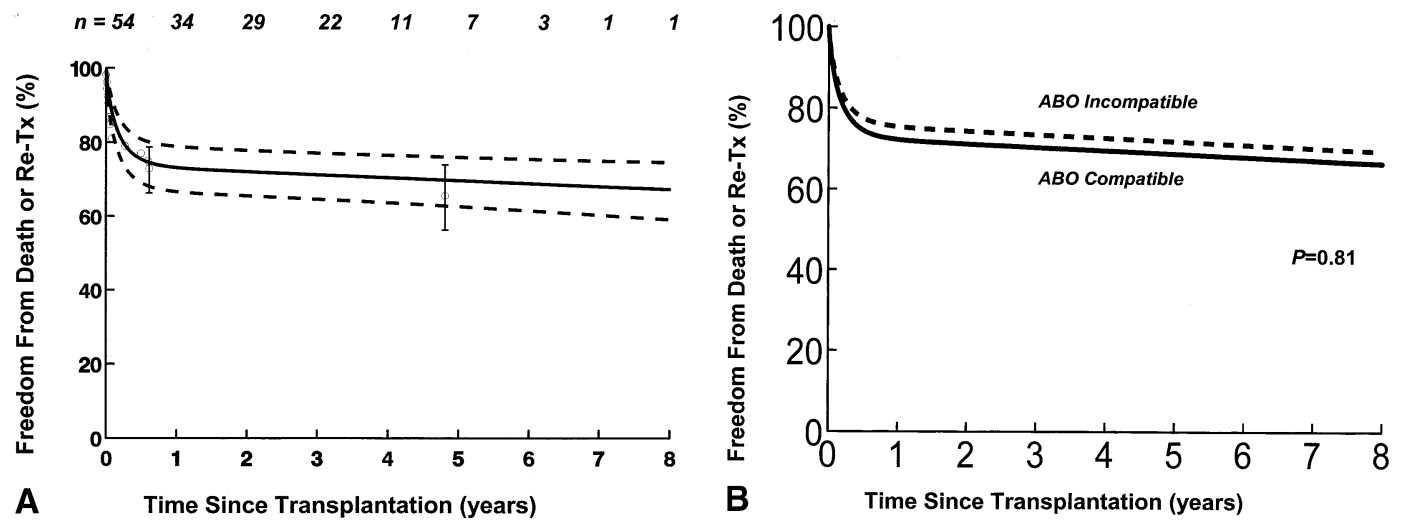

Figure 4. Time-related freedom from outcomes after transplantation. A, Time-related freedom from death or retransplantation after transplantation. Nonparametric Kaplan-Meier estimates (circles with bars). Parametric model of the hazard function (solid line) with $70 \%$ confidence interval (dashed lines). Number of patients at risk (numbers at top of graph). B, Time-related freedom from death or retransplantation after transplantation. Predicted by $\mathrm{ABO}$-compatible versus $\mathrm{ABO}-\mathrm{I}$ transplantation. Prediction for patients receiving an $\mathrm{ABO}-\mathrm{I}$ donor heart (dashed line) versus prediction for patients receiving an $\mathrm{ABO}$-compatible donor heart (solid line). Re-Tx, Retransplantation. 
replacement, and failure to use the ABO-I listing strategy, reflecting the limited donor pool for infants. The only factor found to be significantly associated with achieving transplantation after listing was use of the ABO-I listing strategy. Multivariable competing-risks analysis allowed us to determine the impact of identified risk factors on the rate of transition to each of the 6 defined end states and the proportion of patients achieving each state. We found that adoption of the ABO-I listing strategy resulted in an important decrease in the number of patients who died without transplantation and a corresponding increase in those who underwent transplantation regardless of patient status at listing. Thus, considerable positive clinical impact occurs with the adoption of a prospective ABO-I listing strategy, independent of other factors.

Our data also show that use of an ABO-I strategy did not adversely affect outcomes after transplantation. Specifically, we determined that the risk of either mortality or retransplantation after primary transplantation was not increased by receiving an ABO-I heart graft. Two particular posttransplant problems reported to be associated with antidonor antibodies in adult transplant recipients, acute humoral rejection and transplant vasculopathy, were not specifically examined in this study. However, our data ${ }^{21}$ demonstrating that infant recipients of ABO-compatible heart grafts spontaneously develop specific B-cell tolerance to donor blood group antigens over time suggests that their risk of developing these clinical problems is no greater than for ABO-compatible infant recipients. Moreover, the identical rate of freedom from death and retransplantation between $\mathrm{ABO}-\mathrm{I}$ and $\mathrm{ABO}-$ compatible recipients further confirms that humoral rejection and vasculopathy are likely not of clinically significant difference.

Using an ABO-I strategy for heart transplantation obviously will not generate an increased number of organ donors. However, allocation of heart grafts for infant transplantation unrestricted by the need for $\mathrm{ABO}$ compatibility between donor and recipient could result in redistribution of those rarely available organs of suitable size for infants such that more infants would benefit from transplantation, especially in conjunction with other strategies such as marginal donors, non-heart-beating donors, and geographic matching. Wastage of unplaced donor hearts from the rarer $\mathrm{B}$ and $\mathrm{AB}$ blood group donors, estimated to be up to $60 \%$ of those available, ${ }^{22}$ could be minimized. Furthermore, donor hearts that might require transportation to geographically distant centers to be used for ABO-compatible recipients could be redirected to closer centers. This redistribution would result in decreased graft ischemia times with a concomitant reduction in the associated detrimental effect on posttransplant outcomes. ${ }^{23,24}$

\section{Conclusion}

The adoption of a strategy to accept ABO-I donor hearts for infant transplantation significantly improves the chances of transplantation and reduces death while on the waiting list. In addition, the risks of death or retransplantation after primary transplantation are not adversely affected by receiving an ABO-I heart in this age group. These data continue to support our position that there is no scientific rationale to require $\mathrm{ABO}$ compatibility for infant heart transplantation, and that young recipients with absent or low isohemagglutinin titers should be offered ABO-I transplantation regardless of clinical status.

\section{References}

1. Osorio AV, Sullivan EK, Alexander SR, Bryan CF, Shield CF, Warady BA. ABO-mismatched renal transplantation in children: a report of the North American Pediatric Renal Transplant Cooperative Study (NAPRTCS) and the Midwest Organ Bank (MOB). Pediatr Transplant. 1998;2:26-9.

2. Stock P, Sutherland DE, Fryd DS, Ascher NL, Payne WD, Simmons $\mathrm{RL}$, et al. Detrimental effect of ABO mismatching in renal transplantation. Transplant Proc. 1987;19(1 Pt 1):711-2.

3. Paul LC, Baldwin WM III. Humoral rejection mechanisms and ABO incompatibility in renal transplantation. Transplant Proc. 1987;19(6): 4463-7.

4. Slapak M, Naik RB, Lee HA. Renal transplant in a patient with major donor-recipient blood group incompatibility: reversal of acute rejection by the use of modified plasmapheresis. Transplantation. 1981;31(1):4-7.

5. Bannett AD, Bensinger WI, Raja R, Baquero A, McAlack RF. Immunoadsorption and renal transplant in two patients with a major $\mathrm{ABO}$ incompatibility. Transplantation. 1987;43(6):909-11.

6. Alexandre GP, Squifflet JP, De Bruyere M, Latinne D, Reding R, Gianello P, et al. Present experiences in a series of 26 ABO-incompatible living donor renal allografts. Transplant Proc. 1987;19(6): 4538-42.

7. Sutherland DE, Fryd DS, So SK, Bentley FR, Ascher NL, Simmons RL. Long-term effect of splenectomy versus no splenectomy in renal transplant patients. Reanalysis of a randomized prospective study. Transplantation. 1984;38(6):619-24.

8. Tanabe K, Takahashi K, Sonda K, Agishi T, Kawaguchi H, Ishikawa $\mathrm{N}$, et al. ABO-incompatible living kidney donor transplantation: results and immunological aspects. Transplant Proc. 1995;27(1):1020-3.

9. Gugenheim J, Samuel D, Reynes M, Bismuth H. Liver transplantation across ABO blood group barriers. Lancet. 1990;336(8714):519-23.

10. Eid A, Zamir G, Yaron I, Galun E, Safadi R, Schaaps T, et al. Liver transplantation across the ABO barrier: the role of plasmapheresis. Transplant Proc. 1998;30(3):701-3.

11. Cooper DK. Clinical survey of heart transplantation between ABO blood group-incompatible recipients and donors. J Heart Transplant. 1990;9(4):376-81.

12. West LJ, Pollock-Barziv SM, Dipchand AI, Lee KJ, Cardella CJ, Benson LN, et al. ABO-incompatible heart transplantation in infants. N Engl J Med. 2001;344(11):793-800.

13. McGiffin DC, Naftel DC, Kirklin JK, Morrow WR, Towbin J, Shaddy $\mathrm{R}$, et al. Predicting outcome after listing for heart transplantation in children: comparison of Kaplan-Meier and parametric competing risk analysis. Pediatric Heart Transplant Study Group. J Heart Lung Transplant. 1997;16(7):713-22.

14. Blackstone EH, Naftel DC, Turner ME Jr. The decomposition of time-varying hazard into phases, each incorporating a separate stream of concomitant information. J Am Stat Assoc. 1986;81:615-24.

15. Blackstone EH, Rice TW. Clinical-pathologic conference: use and choice of statistical methods for the clinical study "Superficial adenocarcinoma of the esophagus." J Thorac Cardiovasc Surg. 2001;122:1063-76.

16. Boucek MM, Edwards LB, Keck BM, Trulock EP, Taylor DO, Mohacsi PJ, et al. The Registry of the International Society for Heart and Lung Transplantation: Fifth Official Pediatric Report-2001 to 2002. J Heart Lung Transplant. 2002;21(8):827-40.

17. Chinnock RE, Larsen RL, Emery JR, Bailey LL. Pretransplant risk factors and causes of death or graft loss after heart transplantation 
during early infancy. Pediatric Heart Transplant Team, Loma Linda. Circulation. 1995;92(9 Suppl):II206-9.

18. Morrow WR, Chinnock RE. Survival after heart transplantation. In: Tejani AH, Fine RM, Harmon WE, editors. Pediatric solid organ transplantation. Denmark: Blackwell Publishing; 2000. p 421-2.

19. Morrow WR, Naftel D, Chinnock R, Canter C, Boucek M, Zales V, et al. Outcome of listing for heart transplantation in infants younger than six months: predictors of death and interval to transplantation. The Pediatric Heart Transplantation Study Group. J Heart Lung Transplant. 1997;16(12):1255-66.

20. Ashwal S. Brain death in early infancy. J Heart Lung Transplant. 1993;12(6 Pt 2):S176-S8.
21. Fan X, Ang A, Pollock-Barziv SM, Dipchand AI, Ruiz P, Wilson G, et al. Donor-specific B-cell tolerance after ABO-incompatible infant heart transplantation. Nat Med. 2004;10(11):1227-33.

22. Boucek MM. Breaching the barrier of $\mathrm{ABO}$ incompatibility in heart transplantation for infants. $N$ Engl J Med. 2001;344(11): 843-4.

23. Fleischer KJ, Baumgartner WA. Heart preservation. In: Baumgartner W, Reitz B, Kasper E, Theodore J, editors. Heart and lung transplantation. Philadelphia: WB Saunders Company; 2002. p. 135-41.

24. West LJ. Heart transplantation in children. In: Ginns L, Cosimi A, Morris P, editors. Transplantation. Boston: Blackwell Science Inc.; 1999. p. 485-9. 